\title{
The European VLF/LF radio network: current status
}

\author{
P. F. Biagi · T. Maggipinto - A. Ermini
}

Received: 24 June 2014 / Accepted: 11 November 2014 / Published online: 29 November 2014

(C) Akadémiai Kiadó 2014

\begin{abstract}
For several years researches about correlation between seismicity and disturbances in radio broadcasting are being carried out: in particular, the Japanese Pacific VLF radio network and the European VLF-LF radio network have been developed during the last years. The European network has been developed starting from two LF receivers located in central Italy in 1996. Up to now, 11 receivers of a new type, able to sample the VLF and LF intensity of ten radio signals, are being into operation in different European countries. The daily updating of data is effective and the data bank is located at the Department of Physics of the University of Bari (Italy) which is the central node of the network. In order to discover anomalies, the software able to carry out automatically a daily data analysis by the Wavelet spectra method has been planned and realized. At the moment, the software operates on four signals (two LF and two VLF) collected by one of the receiver located in Italy. If the anomaly is particularly strong a warning system gives an advise on the work station into operation in the central node of the Network. In any case, before assuming an anomaly as a seismic anomaly, geomagnetic and meteorological data must be checked as well as any possible instrumental malfunction. At present these controls are carried out only discontinuously by the researchers of the Bari Team.
\end{abstract}

Keywords Radio signals $\cdot$ Network $\cdot$ Earthquakes $\cdot$ Precursors $\cdot$ Forecast

\section{Introduction}

From several years, a research into the interaction between seismic activity and disturbances in radio broadcasts has been carried out. One of the first results was obtained by using $18 \mathrm{MHz}$ receivers on the occasion of the great $(M=8.5)$ Chilean earthquake of May 22, 1960, but it

P. F. Biagi $(\varangle)$. T. Maggipinto

Department of Physics, University of Bari, Bari, Italy

e-mail: pierfrancesco.biagi@uniba.it

A. Ermini

Department of Industrial Engineering, University of Tor Vergata, Rome, Italy 
was published 22 years later (Warwick et al. 1982). The receivers were part of a network used for studying cosmic noise.

Later, pre-seismic disturbances in VLF radio signals, that lie in the $20-60 \mathrm{kHz}$ frequency band, have been presented mainly by Japanese and Russian researchers (Hayakawa and Sato 1994; Hayakawa et al. 1996, 2002; Morgounov et al. 1994; Molchanov and Hayakawa 1998).

At the same time, pre-seismic disturbances on LF $(150-300 \mathrm{kHz})$ radio broadcasts were proposed mainly by Italian researchers (Bella et al. 1998; Biagi 1999; Biagi et al. 2001a, b; Biagi and Hayakawa 2002; Biagi et al. 2004).

We can call all these disturbances radio precursors. The previous disturbances are related to variations of some parameters in the atmosphere and ionosphere, i.e. in the propagation medium of the radio signals. Two different models have been proposed to justify the atmospheric and ionospheric disturbances.

The first one assumes a direct effect, that is a ionizing radiation due to gases (mainly radon)/aerosol or electromagnetic emissions coming from the crust during the preparatory phase of an earthquake (Alperovitch 1997; Biagi 1999; Biagi et al. 2001b; Hayakawa and Sato 1994; Pulinets et al. 1998).

The second model assumes an indirect effect, that is the production of (AGW) atmospheric gravity waves (Hayakawa et al. 1996; Molchanov and Hayakawa 1998) in the atmosphere due to changes of local gravity connected with pre-seismic micro-fracturing processes and ground uplift and tilt. The second model overcomes the problem in the first model of the transport up to ionosphere of particles or electromagnetic waves from the ground. In any case the atmospheric and radio precursors indicate the existence of an ionosphere-atmospherelithosphere coupling.

The VLF radio signals are used for time signal, navigation and military purposes and they propagate using the Earth-ionosphere channel as waveguide.

The LF radio signals are used in long wave broadcasting and are characterized by a groundwave and a sky-wave propagation mode. The first generates a stable signal that propagates in the channel Earth-troposphere; whereas the second generates a signal which varies greatly between day and night, summer and winter, and which propagates using the lower ionosphere as a reflector.

Generally, the radio data used in the research on radio precursors are collected by receivers located on the ground. Recently, also the use of satellites for this purpose has been planned and achieved (Molchanov et al. 2006).

\section{Radio receivers and ground networks}

Since 2000 Japanese researchers have been relying on the existence of a Pacific VLF radio network of seven receivers able to measure, with a sampling rate of $20 \mathrm{~s}$, the intensity and the phase of VLF radio signals radiated by two transmitters (Fig. 1a). The receivers are the Japanese OmniPal model (Dowden and Adams 1989) and are connected to a computer through a digital card and use a GPS sensor and a rectilinear antenna for the signal's registration (Fig. 1b).

During 1996, a receiver able to measure the intensity of three LF radio signals, with a sampling rate of $10 \mathrm{~min}$, was planned and realized by Italian researchers. Two equipments were put into operation in sites located in the Apennines (central Italy). On February 2002, in the framework of a scientific cooperation among Japanese, Russian and Italian teams, a Japanese OmniPal receiver, was put into operation in the Department of Physics of the University of Bari (south Italy). In such a way the development of a European radio network 

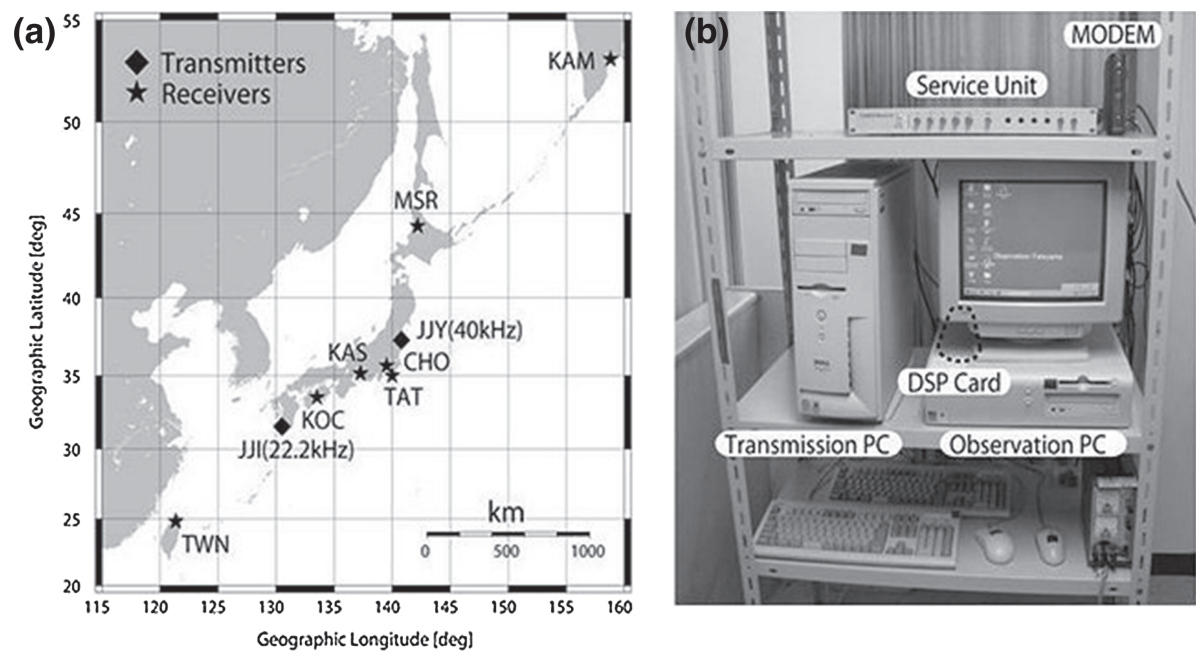

Fig. 1 a The Pacific VLF radio network. The receiver (OmniPal) are marked by stars and are located five in Japan, one in Kamchatka (Russia) and one in Taiwan. The signals collected come from JJY (40 kHz) and JJI $(22.2 \mathrm{kHz})$ transmitters located in Japan. b The OmniPal recording system
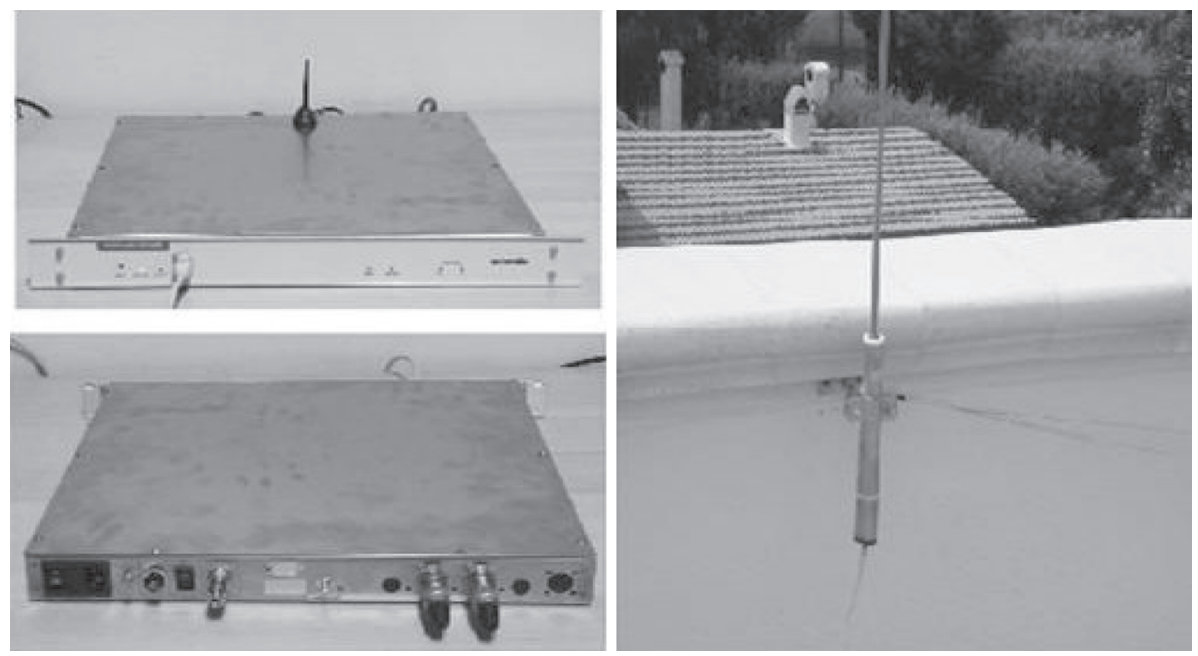

Fig. 2 On the left the Elettronika receiver (front and back side) is shown; on the right one antenna with its preamplifier

starts. During 2008 a new receiver was developed by the Italian factory Elettronika (Palo del Colle/Bari). The new receiver, shown in Fig. 2, can work both in the VLF and the LF band. It can monitor ten frequencies in all distributed in these bands and, for each of them, saves the power level (intensity of the signal) detected on a big non-volatile memory at a selectable sample time interval. The receiver has two standard XLR antenna connectors, one for each band, with four poles. On the same connector there is the differential input signal from the antenna and the dual power supply voltage for the preamplifier that is located near the antenna. Its aim is to convert the high impedance of the low-frequency antennas in a low impedance to drive the cable and to amplify the captured signal level introducing very low noise. 


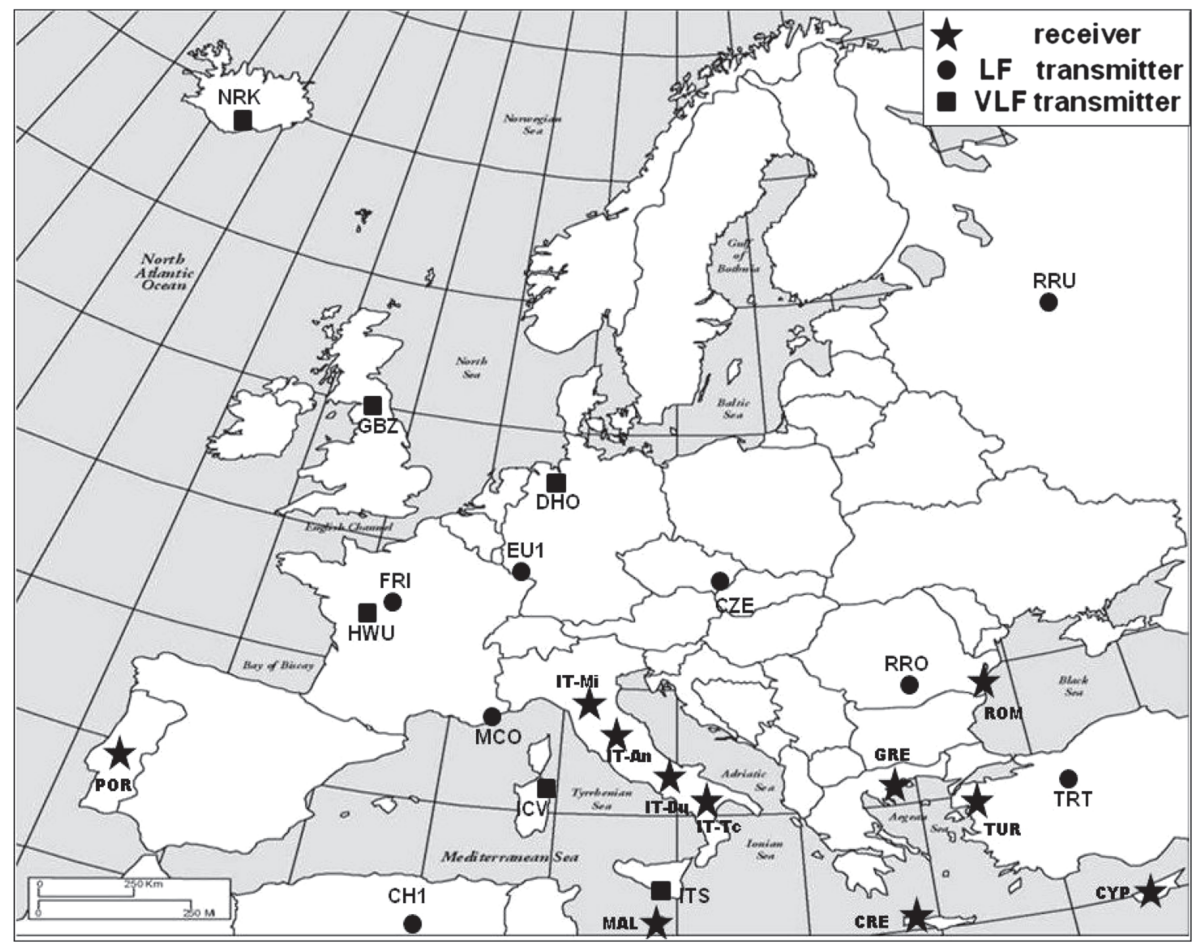

Fig. 3 Map showing the receivers and transmitters of the network

The two antennas, made of brass, are rectilinear ones, long $1.5 \mathrm{~m}$ (LF band) and $2.0 \mathrm{~m}$ (VLF band), respectively. The huge amount of data collected is organized in text files, one for each day. The configuration of the equipment, the check of its status and the download of the collected data are possible by means of a resident software with a web based graphical user interface and of an HTTP server. Data transfer and management is also possible through a resident FTP server. Then, either local, either remote TCP/IP connectivity with the instrument and above mentioned software is allowed by means of a standard ethernet interface and of a GSM modem (configured for data calls) both mounted in the chassis of the receiver. It should be mentioned that the receiver was designed by requiring the ease of interactivity and communication. Thanks to the facility of remote and furthermore "web-ready" data management, the set up of a network of such receivers, located in strategic sites (from a seismic point of view), was straightforward.

During 2009-2011 11 receivers were prepared, four for Italy and one for Cyprus, Crete, Greece, Malta, Portugal, Romania and Turkey. At the moment the installation of the different receivers is still not complete.

The European VLF/LF radio network is shown in Fig. 3. A sampling rate of $1 \mathrm{~min}$ is actually used. Some peculiarities of the transmitters are reported in Table 1.

\section{Data analysis}

In order to discover disturbances in the radio data, suitable techniques of analysis must be applied. 
Table 1 Peculiarities of the transmitters of the European VLF/LF radio network

\begin{tabular}{llll}
\hline Label & Country & Power $(\mathrm{kW})$ & Frequency $(\mathrm{kHz})$ \\
\hline GBZ & Great Britain & & $19.58-22.10$ \\
ICV & Sardinia (Italy) & & 20.27 \\
HWU & France & & $20.90-21.75$ \\
DHO & Germany & & 23.4 \\
NRK & Iceland & & 37.5 \\
ITS & Sicily (Italy) & 1,200 & 45.9 \\
RRO & Romania & 2,000 & 153 \\
FRI & France & 2,000 & 162 \\
EU1 & Germany & 2,000 & 183 \\
CH1 & Algeria & 1,200 & 198 \\
MCO & France & 2,500 & 216 \\
RRU & Russia & 500 & 261 \\
CZE & Czech Republic & 270 \\
\hline
\end{tabular}

It must be taken into account that at day time, the VLF/LF signals are strongly disturbed; so, generally the analyses are conducted only on the night data, while only sometimes the day data are analyzed.

\subsection{Pacific VLF radio network}

The techniques of analysis TT (terminator times) and $A, P$ (amplitude, phase) fluctuations are used. The first one is based on terminator time which is defined as the time when the diurnal amplitude (or phase) variation of the signal exhibits a minimum around sunrise and sunset; so morning ( $\mathrm{tm}$ ) and evening (te) terminator times can be defined. The shifts of $\mathrm{tm}$ to early time and of te to later time with respect to unperturbed situations represent the TT anomalies (Molchanov and Hayakawa 1998)

The second technique (Rozhnoi et al. 2005, 2006a) uses a residual signal of amplitude $d A$ or phase $d P$, defined as the difference between the signal and the average of few quiet days ( \pm 5 days) immediately preceding or following the current day: $d A=A-\langle A\rangle$ and $d P=P-\langle P\rangle$ where $A$ and $P$ are the mean amplitude and the mean phase for the current day (night), while $\langle A\rangle$ and $\langle P\rangle$ are the averages of the mean values on the quiet days (nights). A day is considered quiet in absence of ionosphere or magnetosphere activity, of magnetic storms, of atmosphere cyclones and so on (Sect. 4).

\subsection{European VLF/LF radio network}

The techniques of analysis Wavelet spectra and standard deviation $(S D)$ trends are used.

The Wavelet transform allows to highlight the spectral components of a signal by using variable-width time windows and by considering that the frequency content of these windows is in inverse relation to the time widths; so, the localization of the signal is simultaneously obtained both in time and in frequency. The "Morlet function" is adopted as Wavelet and, in this case, the Wavelet transform of a time signal is a complex series that can be usefully represented by its square amplitude, i.e. we consider the so-called Wavelet power spectrum. The power spectrum is a two dimensions plot that, once properly normalized with respect to 
the power of the white noise, gives information on the strength and precise time of occurrence of the various Fourier components which are present in the original time series. Generally, in a color illustration, colour from blue to red indicates increase in the power strength; so, red zones define anomalies (Biagi et al. 2006, 2008).

The second technique of analysis is based on the $S D$. For a sample of $n$ data, the $S D$ is calculated as follows:

$$
S D=\sqrt{\frac{\sum_{1}^{n}\left(x_{i}-x_{m}\right)^{2}}{n-1}}
$$

where $x_{\mathrm{m}}$ is the mean value of the $x_{\mathrm{i}}$ values. $S D$ shows how much variation or "dispersion" there is from the mean. A low $S D$ value indicates that the data points tend to be very close to the mean, whereas high $S D$ value indicates that the data points are spread out over a large range of values. The $S D$ can be calculated for each set of data and particularly low/high values define anomalies, that is low or high dispersion of the data. For a larger evidence the $\%$ value is used, which is defined as $\left(S D-S D_{\mathrm{m}}\right) / S D_{\mathrm{m}}$ where $S D$ is the value of each day/night-time data and $S D_{\mathrm{m}}$ is the mean value of the $S D$ data set in the whole time interval analyzed. The related trends are the $S D$ trends and the values over $\pm 2 \sigma(S D)$ in these trends are assumed as low/high values defining an anomaly (Righetti et al. 2012).

\section{Disturbances in VLF/LF radio signals}

The research of seismic effects on the VLF/LF radio signals is based on the spotting of disturbances on the data. In this framework, a first and fundamental step is the identification of possible disturbances related to causes different from the seismicity.

The meteorological conditions and the geomagnetic activity can affect the propagation of VLF/LF radio signals. Particularly, the meteorological conditions produce disturbances in the troposphere refractive index, troposphere electricity (lightning), the relative permittivity and the conductivity of the ground; the geomagnetic activity produces variations in the ionosphere conditions. Disturbances on the propagation of the VLF/LF radio signals related to these effects have been presented (Kikuchi 1981; Kleimenova et al. 2004; Biagi et al. 2005; Rozhnoi et al. 2006a, b). In Biagi et al. (2011), it was revealed that the geomagnetic activity affects mainly the VLF radio propagation while the meteorology (conditions in the transmission-reception zones) mainly the LF radio signals. Anyway, if a correlation is claimed between radio disturbances and the meteorological/geomagnetic variations, they must be contemporary. So, the contemporaneity is one of the criteria to individuate these radio disturbances.

In addition, the study mentioned above has revealed disturbances in radio signals related to variations of the power radiated by the transmitters, to some breaking of the receiver and to some human activity at a receiver site. The LF radio signals seem more disturbed than the VLF ones. If the effect is due to the transmitter, the disturbance must appear simultaneously in the related signal recorded by the different receivers of the network; if the effect is due to a receiver, disturbances must appear simultaneously in all the signals collected by this receiver So, these types of radio disturbance can be identified with a strict analysis of the data collected. If the disturbances revealed in the radio data have get over the previous steps, the possibility of a pre-seismic effect becomes realistic. 


\section{$5 \mathrm{VLF} / \mathrm{LF}$ radio precursors and the earthquake forecast}

In order to use the VLF/LF radio signals anomalies for earthquake forecast it is necessary:

(a) a daily updating of the data collected by the radio receivers of the network;

(b) a daily study of the data using the methods of analysis reported in Sect. 3 in order to discover possible anomalies;

(c) a simultaneous control of causes different from the seismicity (Sect. 4) related to the previous anomalies.

\subsection{Pacific VLF radio network: state-of-the-art}

As concerns the Pacific VLF radio network, the collected data are used in a system of earthquakes forecast actually active in Japan. This service is done for a free by a private company [Hayakawa Institute of Seismo Electromagnetics Co. Ltd., EAL (Earthquake Analysis Laboratory)], managed by M. Hayakawa, professor emeritus at the University of ElectroCommunication in Tokyo and founder of the previous network. The presentation of the company is: "the Earthquake Analysis Laboratory is doing a service of providing the public with the earthquake predictive information based on the measurements of VLF/LF ionospheric perturbations, ULF electromagnetic emissions and GPS satellite total electron contents. The practical service of releasing the earthquake forecast information to the public is the first attempt in the world, and this service was started since April, 2011. We haven't detailed information regarding the methodology used; in any case several forecasts of strong $\left(\mathrm{M}_{\mathrm{w}} \geq 6.5\right)$ earthquakes have been claimed in the last 3 years as it is reported (in Japanese) in the web site: http://www.earthquakenet.com.

\subsection{European radio network: state-of-the-art}

The central node of the Network is the Department of Physics of the University of Bari (Italy); here the work station assigned to the Network is located. The daily updating of data is effective and the temporal intensity trends of the different signals collected by the Network receivers can be seen at INFREP web site (http://beta.fisica.uniba.it/infrep/Home. aspx). Here, at first, clicking on each receiver the 5th Fresnel zones of the collected signals are shown (Fig. 4). Then, clicking on CHARTS (radio data) it is possible to select the receiver and the time interval required (Fig. 5a); finally, the trends of the ten radio signals will come out. In Fig. $5 \mathrm{~b}$ an example of a part of trends is reported. Inside CHART it is possible also to see the temporal trend of $K_{\mathrm{p}}$ and $D_{\text {st }}$ geomagnetic indices and the meteorological data of some receiver site.

The data bank (updated in real time) is accessible by web site clicking on login on the home page and using username and password; at present only the responsible of each receiver can enter the bank and download the data.

An example of radio data set is reported in Fig. 6.

Among the two methods of analysis described in Sect. 3.2 the Wavelet one has been selected and the software able to apply the analysis on the radio data automatically at the end of each day has been planned and realized.

At the moment this software operates on the night time ( $3 \mathrm{~h}$ ) data of four signals (Fig. 7a) collected by one of the Italian receivers (IT-Tc).

On Fig. 7b, as an example, the two dimensions Wavelet Spectrum of one of these signals ( $162 \mathrm{kHz}$-France) is shown. The analysis is performed on the 20 days preceding September 


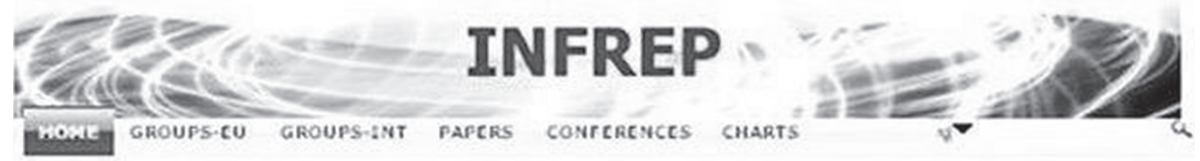

\section{International Network for Frontier Research on Earthquake Precursors}

INFREP is a scientific cooperation among different International research groups. The cooperation aims to build up a network for measuring various physical parameters in onder to search and study effects possibly related to the occurrence of earthquakes.

In particular the first network consists of several receiver able to measure VUF. LF radio signal from warious broadcasting stations located throughout Europe.

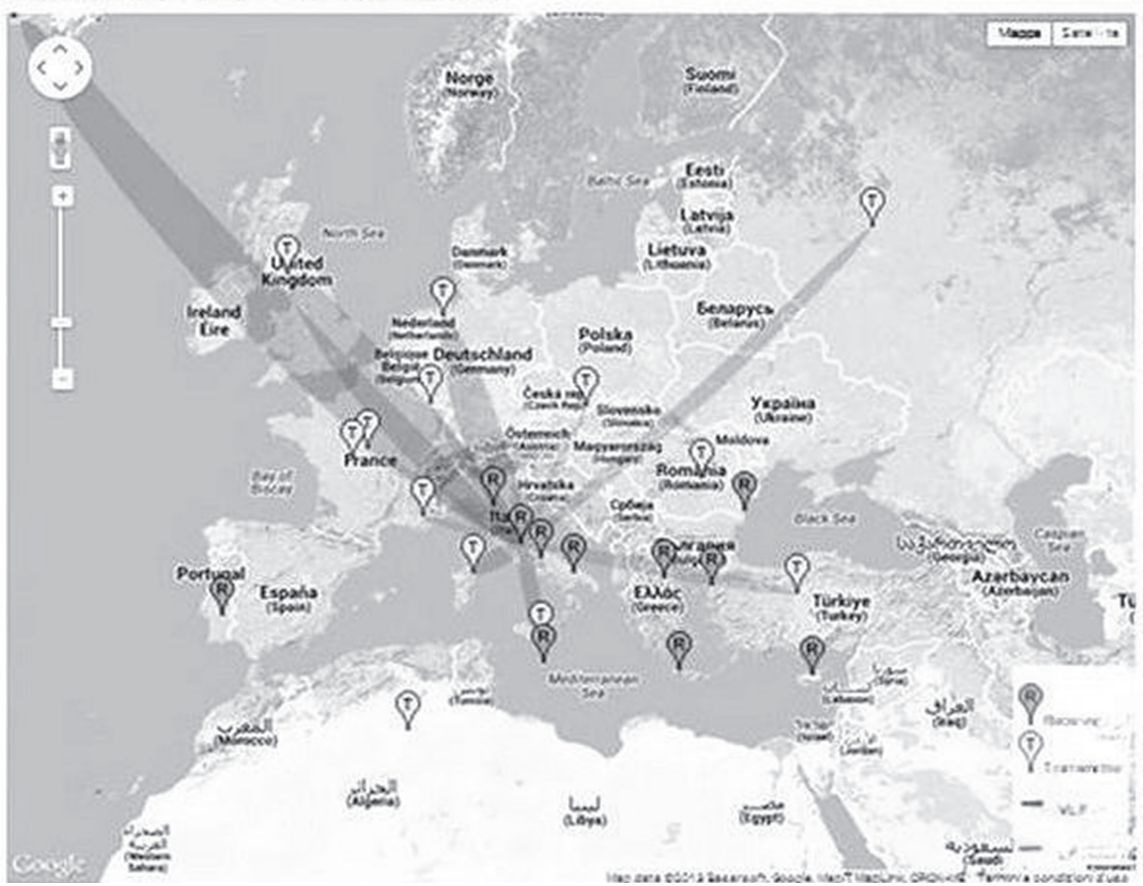

Fig. 4 The ellipses represent the 5th Fresnel zones of the radio signals sampled by the IT-An receiver of the Network

9, 2013; this day is indicated by the vertical white line. The right part of the spectrum is related to 15 days data without any frequency, added to avoid border effects.

On the basis of the results obtained in previous studies (Hayakawa et al. 2002; Biagi et al. 2006, 2011; Righetti et al. 2012) a threshold level for the size of the radio anomaly was fixed.

When an anomaly over this level is revealed in one of the four mentioned signals a warning message appears on the work station of Bari. One of such situations is shown in Fig. 8. The control of the meteorological condition along the path of the signal has allowed to consider realistic the connection of this anomaly with the large storm occurred in that period in the north area of Adriatic sea. 


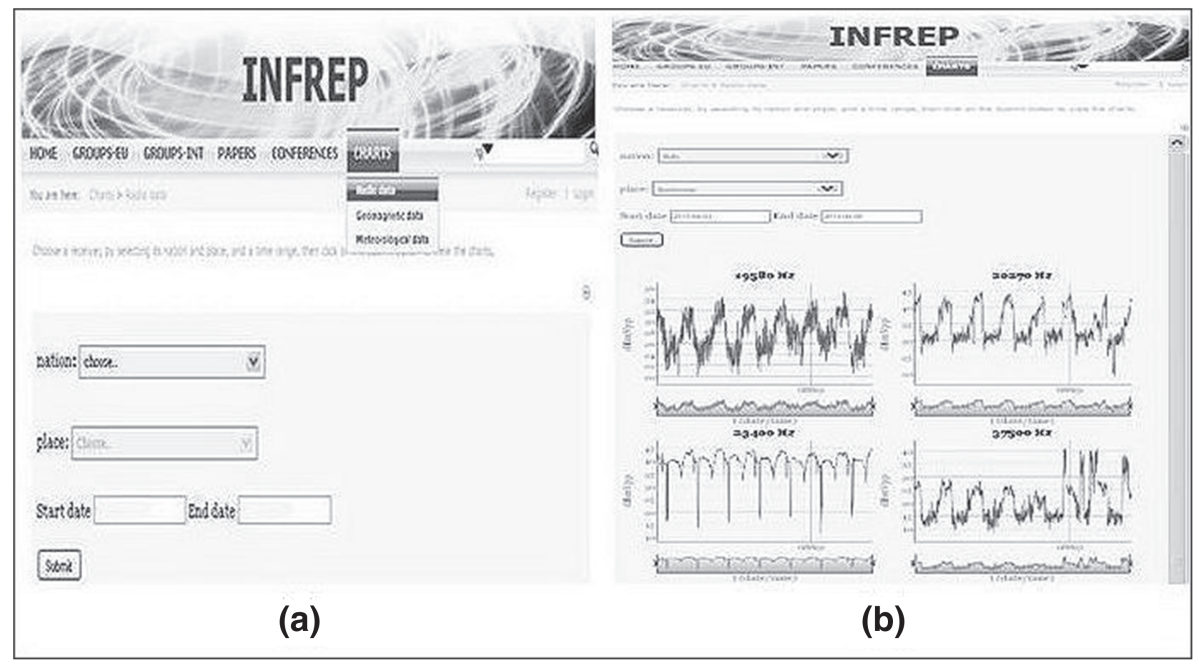

Fig. 5 a The panel appearing after clicking on CHART (radio data) where it is possible to select the receiver (nation and place) and the time interval required; b part of the trends selected with the previous actions

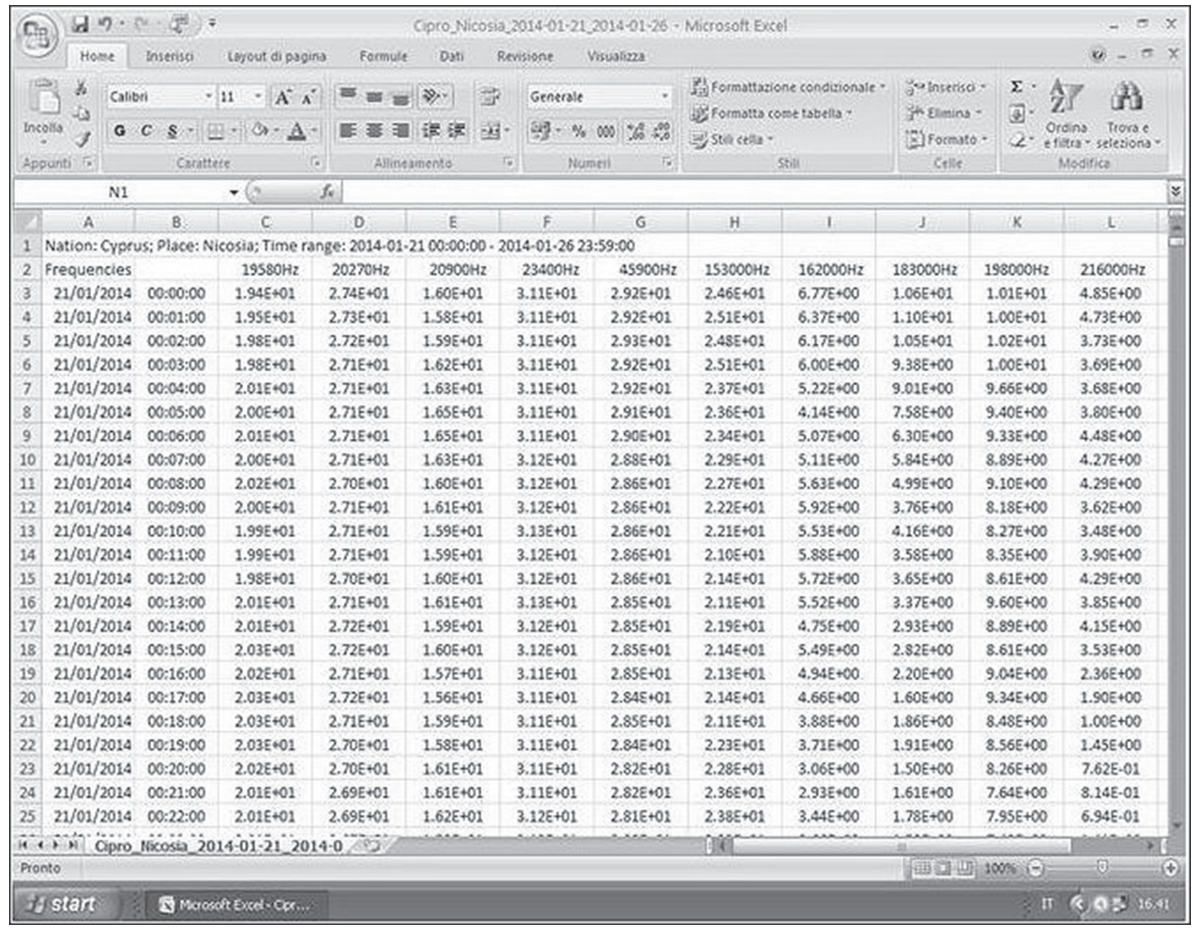

Fig. 6 Data set of the ten radio signals collected by the receiver in Cyprus on January 21, 2014 from 00.00 to $00.22 \mathrm{UT}$

The control of causes different from the seismicity related to the radio anomalies is a crucial point in our research. We are trying to realize a software able to make this control automatically, but it is very difficult because too many factors must be examined. Probably, 


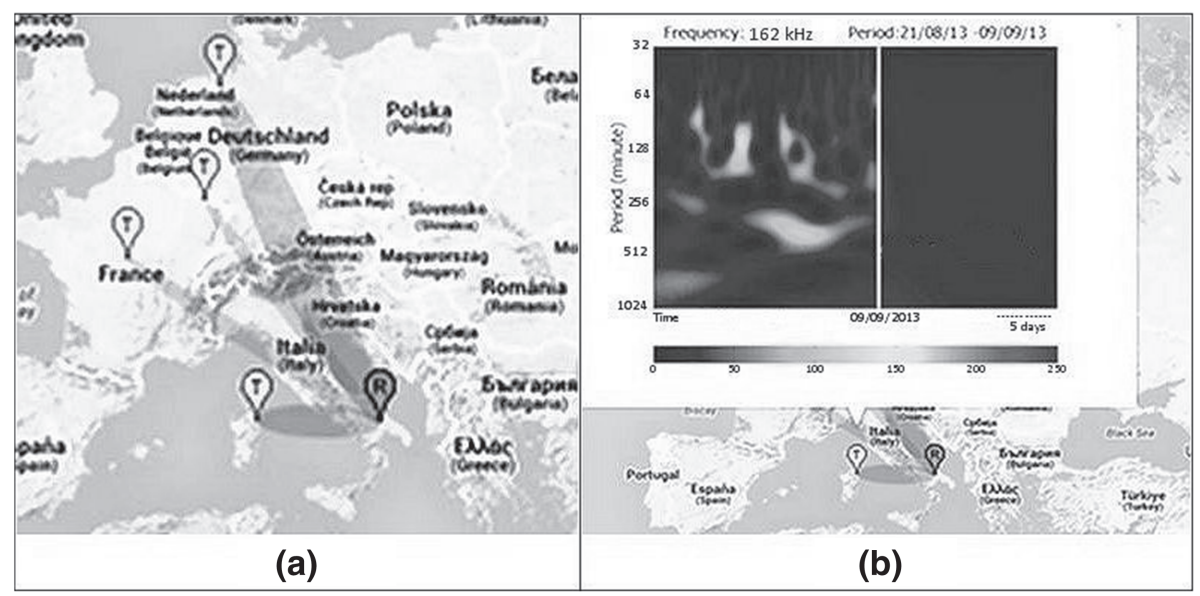

Fig. 7 a The 5th Fresnel zones of the four signals collected by the IT-Tc receiver which are daily analyzed using the Wavelet method; $\mathbf{b}$ the two dimensions Wavelet Spectrum of the $162 \mathrm{kHz}$ signal obtained at September 10, 2013; this day is indicated on the Spectrum by the vertical line

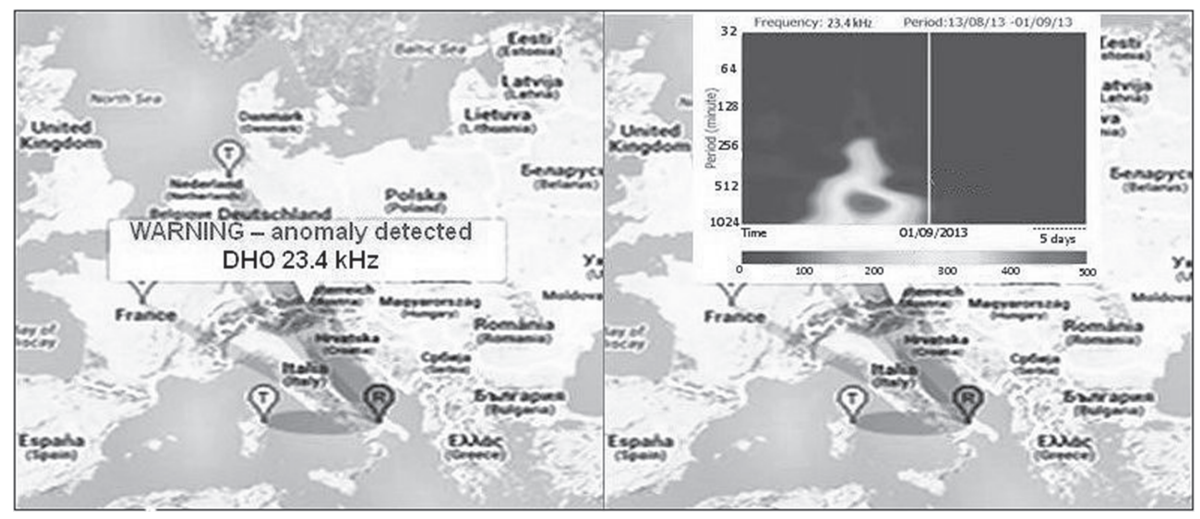

Fig. 8 Warning alert appeared at the beginning of September 2013 on the $23.4 \mathrm{kHz}$ radio signal recorded by the IT-Tc receiver

the software will be able to exclude some factor, but a complete scanning cannot be carried out without the check of some researcher expert in this field.

The choice of the threshold level of the Wavelet anomaly is another crucial point in our research. In fact, in some case the size of the seismic anomaly is not very large; so, a lower value of the threshold should be adopted. But, in such way the warning message could appear frequently in the signals and the anomaly could remain also after the control procedure. So, several false alarms could occur.

\section{Conclusions}

In order to study the VLF/LF radio precursors of earthquakes, a network is into operation in Europe. The data collection is online and the data are updated each day. The software able to reveal Wavelet anomalies in four radio signals collected by one receiver of the network has been set up. The technique can be extended to other signals and to other receivers. It must be taken into account that an anomaly in the radio signals can be produced by several causes 
different from seismicity; therefore controls must be carried out before to claim a possible precursor of earthquakes.

The European VLF/LF radio network is a good step for producing a forecast of some strong earthquake, but in order to have a satisfactory probability of success, it is necessary to have at disposal simultaneously the warning advices coming from several other different parameters.

\section{References}

Alperovitch L (1997) Perturbation of atmospheric conductivity as a cause of the seismo-ionospheric interaction. Ann Geophys 15, 1, Abstract - XXII EGS Gen. Assembly, Vienna, Austria, April 1997

Bella F, Biagi PF, Caputo M, Cozzi E, Della Monica G, Ermini A, Plastino W, Sgrigna V (1998) Field strength variations of LF radio waves prior to earthquakes in Central Italy. Phys Earth Planet Inter 105:279-286

Biagi PF (1999) Seismic effects on LF radiowaves. In: Hayakawa M (ed) Atmospheric and ionospheric electromagnetic phenomena associated with earthquakes. TERRAPUB, Tokyo, pp 535-542

Biagi PF, Piccolo R, Ermini A, Martellucci S, Bellecci C, Hayakawa M, Capozzi V, Kingsley SP (2001a) Possible earthquake precursors revealed by LF radio signals. Nat Hazards Earth Syst Sci 1:1-2

Biagi PF, Piccolo R, Ermini A, Martellucci S, Bellecci C, Hayakawa M, Kingsley SP (2001b) Disturbances in LF radio-signals as seismic precursors. Ann Geofis 44(5/6):1011

Biagi PF, Hayakawa M (2002) Possible premonitory behaviour of LF radiowaves on the occasion of the Slovenia earthquakes (M= 5.2-6.0-5.1) occurred on March-May 1998. In: Hayakawa M, Molchanov O (eds) Seismo electromagnetics: lithosphere-atmosphere-ionosphere coupling. TERRAPUB, Tokyo, pp 249-253

Biagi PF, Piccolo R, Castellana L, Ermini A, Martellucci S, Bellecci C, Capozzi V, Perna G, Molchanov O, Hayakawa M (2004) Variations in a LF radio signal on the occasion of the recent seismic and volcanic activity in Southern Italy. Phys Chem Earth 29(4/9):551-557

Biagi PF, Maggipinto T, Ermini A et al (2005) A possible preseismic anomaly in the ground wave of a radio broadcasting (216 kHz) during July-August 1998 (Italy). Nat Hazards Earth Syst Sci 5:727-732

Biagi PF, Maggipinto T, Ermini A et al (2006) LF radio anomalies revealed in Italy by the wavelet analysis: possibile preseismic effects during 1997-1998. Phys Chem Earth 31:403-408

Biagi PF, Maggipinto T, Ermini A et al (2008) Disturbances in a VLF radio signal prior the M=4.7 offshore Anzio (central Italy) earthquake on August 22, 2005. Nat Hazards Earth Syst Sci 8:1041-1048

Biagi PF, Maggipinto T, Ermini A et al (2011) The European VLF/LF radio network to search for earthquake precursors: setting up and natural/man-made disturbances. Nat Hazards Earth Syst Sci 11:333-341

Dowden RL, Adams CD (1989) Phase and amplitude perturbations on the NWC signal at Dunedin form lightning-induced electron precipitation. J Geophys Res 94:497-503

Hayakawa M, Sato H (1994) Ionospheric perturbations associated with earthquakes, as detected by subionospheric VLF propagation. In: Hayakawa M, Fujinawa Y (eds) Electromagnetic phenomena related to earthquake prediction. TERRAPUB, Tokyo, pp 391-397

Hayakawa M, Molchanov OA, Ondoh T, Kawai E (1996) Anomalies in the subionospheric VLF signals for the 1995 Hyogo-ken earthquake. J Phys Earth 44:413-418

Hayakawa M, Molchanov OA, Shima N, Shvets AV, Yamamoto N (2002) Wavelet analysis of disturbances in subionospheric VLF propagation correlated with earthquakes. In: Hayakawa M, Molchanov O (eds) Seismo electromagnetics: lithosphere-atmosphere-ionosphere coupling. TERRAPUB, Tokyo, pp 223-228

Kikuchi T (1981) VLF phase anomalies associated with substorm. Mem Natl Inst Polar Res (Spec Issue) $18: 3-23$

Kleimenova NG, Kozyreva OV, Rozhnoi AA, Solovieva MS (2004) Variation in the VLF signal parameters on the Australia-Kamchatka radio path during magnetic storms. Geomagn Aeron 44(3):354-361

Molchanov OA, Hayakawa M (1998) Subionospheric VLF signal perturbations possibly related to earthquakes. J Geophys Res 103:17.489-17.504

Molchanov OA, Rozhnoi A, Solovieva M, Akentieva O, Berthelier JJ, Parrot M, Lefeuvre F, Biagi PF, Castellana L, Hayakawa M (2006) Global diagnostic of the ionospheric perturbations related to the seismic activity using the VLF radio-signals collected on the DEMETER satellite. Nat Hazards Earth Syst Sci 6:745-753

Morgounov VA, Ondoh T, Nagai S (1994) Anomalous variation of VLF signals associated with strong earthquakes $(M \geq 7.0)$. In: Hayakawa M, Fujinawa $Y$ (eds) Electromagnetic phenomena related to earthquake prediction. TERRAPUB, Tokyo, pp 409-428 
Pulinets SA, Hegai VV, Boyarchuk KA, Alekseev VA (1998) The new conception od earthquake prediction. Ann Geophys. 16, 1, Abstract - XXIII EGS Gen. Assembly, Nice, France, April 1998

Righetti F, Biagi PF, Maggipinto T, Ermini A et al (2012) Wavelet analysis of the LF radio signals collected by the European VLF/LF network from July 2009 to April 2011. Ann Geophys 55(1):171-180. doi:10. 4401/ag-5188

Rozhnoi AA, Solovieva MS, Molchanov OA, Hayakawa M, Maekawa S, Biagi PF (2005) Anomalies of LF signal during seismic activity in November-December 2004. Nat Hazards Earth Syst Sci 5:657-660

Rozhnoi AA, Solovieva MS, Molchanov OA, Chebrov V, Voropaev V, Hayakawa M, Maekawa S, Biagi PF (2006a) Preseismic anomaly of LF signal on the wave path Japan-Kamchatka during November 2004. Phys Chem Earth 31:422-427

Rozhnoi A, Solovieva MS, Molchanov OA, Hayakawa M, Maekawa S, Biagi PF (2006b) Sensitivity of LF signal to global ionosphere and atmosphere perturbations in the network of stations. Phys Chem Earth 31:409-415

Warwick JW, Stoker C (1982) Radio emission associated with rock fracture: possible application for the Great Chilean Earthquake of May 22, 1960. J Geophys Res 87:2851-2859 